\title{
Anacardic Acid Derivatives as Potential Acetylcholinesterase Inhibitors
}

\author{
A. S. Kiametis, M. Abreu, R. Gargano \& L. A. S. Romeiro
}

\section{Introduction}

Alzheimer's disease is the leading cause of dementia among people over 65 years of age. Although its etiology is not fully known, the decreased levels of acetylcholine (ACh) has been linked to the pathophysiology of the disease [1]. The cholinergic hypothesis is a line therapy based on increasing the level of acetylcholine by reversible inhibition of the enzyme acetylcholinesterase (AChE) therefore promoting an improvement in the patient's cognitive profile. This paper aims to propose possible candidates to $\mathrm{AChE}$ inhibitors, designed from the lipid phenolic derivatives of cashew, more specifically the anacardic acid, the most abundant phenolic lipid derivative extracted from the cashew nutshell liquid, which is a natural resin found in the honeycomb structure of the cashew (Anacardium occidentale) nutshell.

\section{Methodos}

AChE structure looks like a deep narrow gorge (figure 1), with about 20A length, lined by 14 conserved aromatic residues [2]. Among these residues, several functional amino acids have been identified such those in the catalytic triad where AChE hydrolyses ACh. After being recognized by the residues localized among the peripheral anionic site and the aromatic site (a hydrophobic subunit), ACh reaches deep into the protein binding to the catalytic triad and the aromatic site. The ACh's amino group binds to the aromatic Trp 84 by cation- $\pi$ interactions while Tyr70, Tyr121 and Phe330 residues act on $\mathrm{ACh}$ through hydrophobic-aromatic interactions with methylene spacers containing up to 8 carbons.

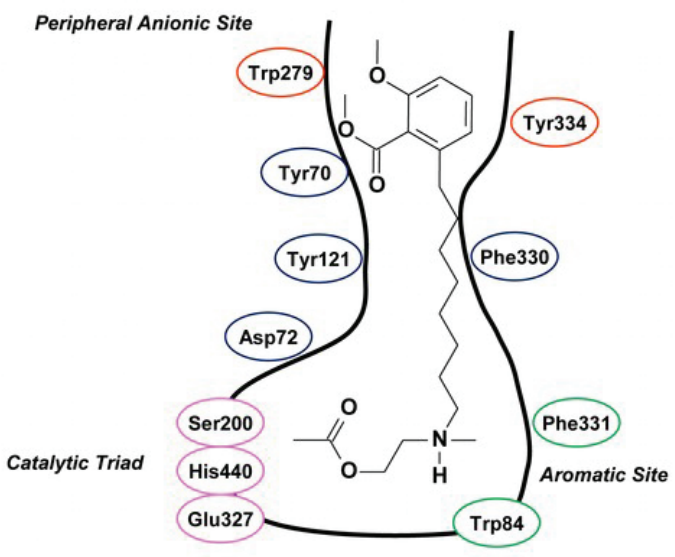

Figure 1. Acetylcholinesterase - a schematic representation of the inhibitor candidate interaction with the residues of elctrophorus electricus AChE. 
Our compounds were designed to mimics ACh behavior so they were divided in two pharmacophoric subunits (figure 2), according to the respectively site in which may occur a interaction (peripheral or catalytic). A hydrophobic spacer sufficiently long was inserted between the two subunits thus the drug occupies the whole gorge and the expected interactions becomes possible.

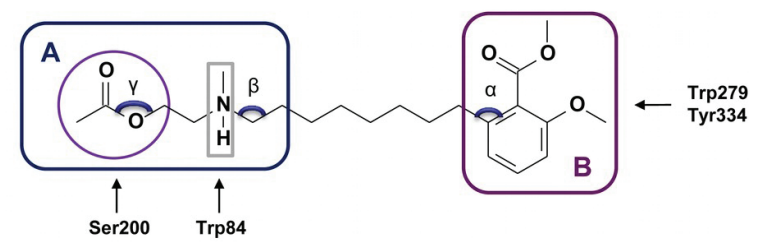

Figure 2. Design of our inhibitor candidates of AChE.

In total, twenty molecular structures with different functional groups were designed taking into account the possible interactions between these functional groups and enzyme active sites. For each molecule, a conformational analysis was done in a way to identify the more stable conformers. The geometries were first optimized by semiempirical method and then optimized again, for greater accuracy, by using the hybrid functional B3LYP and and basis set 6- $311+\mathrm{G}(2 \mathrm{~d}, \mathrm{p})$. A single point was taken for the equilibrium geometry for each compound and several electronic properties important for the molecular recognition by the enzyme, like HOMO, HOMO-1, LUMO, LUMO+1, GAP and atomic charges, were calculated for the compounds studied. The polarizable continuum model has been implemented in the ab initio calculations to simulate solvating. Geometric and lipophilicity properties were also determined for each candidate using quantitative structure activity relationship

\section{Results and Discussion}

The descriptors for this group of molecules are strongly correlated so we have used the Principal Component Analysis (PCA) to treat the initial data set. This technique has been successfully used before to predict the most relevant descriptors to correlate inhibitors and active drugs
[3]. Here, we tried to correlate our candidates with donpezil. To ensure accurate results, the PCA was performed taking into account all possible linear combinations of the variables (descriptors). For a cluster analysis, only those combinations in which the PC1 variance showed equal or greater than $75 \%$ were selected. After systematic analysis, for the case where the PCM solvating model was not used in electronic structure calculations, in general, the molecules number 5 and 7 remained near the drug for the first two principal components. Others like number 1-10, 14 and 16 remained near the drug for PC1 but not for PC2. The descriptors identified as the most suitable for the correlation are Mulliken charge of the nitrogen belonging to amino group $(\mathrm{N}), \log \mathrm{P}$, polarizability and molecular mass, whereas the latter two contribute more effectively to the PC1, accounting $79 \%$ of the information about the correlation. Including the PCM solvating model in electronic structure calculations, the distribution of molecules in the first two principal components is expressively affected. In this situation, molecules 5 and 7 still remain close to donepezil for PC1 but deviate considerably for PC2. Molecule 16, which was far from the drug for PC2, now is the most correlated with donepezil, according to figure 6 . The score plot of PC1 versus PC2 (figure 3) shows how remarkably close molecule 16 is to donepezil for both components. The acid character of the carboxyl group of the anacardic acid is possibly influencing the ligand-receptor interactions in the presence of solvent, leading to this result.

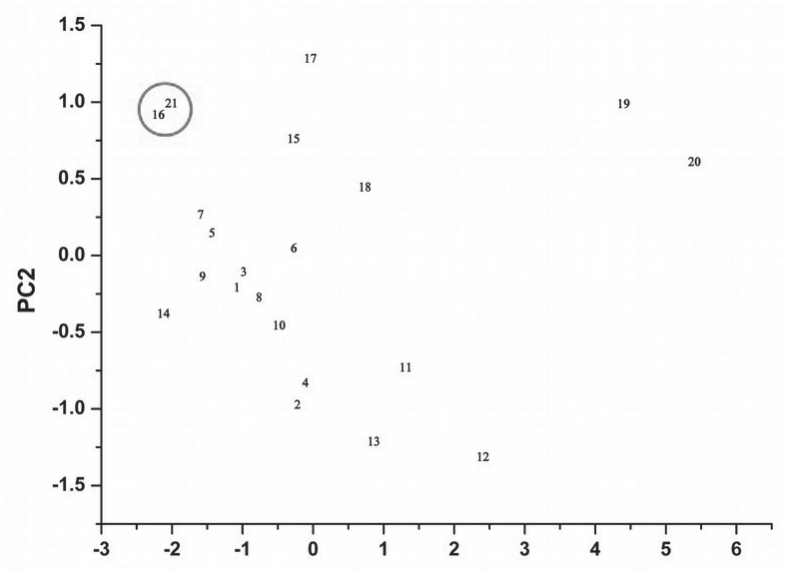

Figure 3. Score plot of PC1 versus $\mathrm{PC} 2$ in a solvating model. The clusterinvolving donepezil and molecule 16 is shown. 
In structural terms, molecule 16 is more similar to donepezil than the others. The molecule has a benzene heterocyclic ring bonded to an amine group where is concentrated the HOMO orbital, showing the electron donor character of the compound in this region. The HOMO energy for molecule 16 was $-8.797 \mathrm{eV}$. In this case, the benzene group is possibly interacting with $\operatorname{Trp} 84$ residue through cation- $\pi$. The LUMO orbital is concentrated at the dimethoxy-phenyl ring, showing the electron acceptor character of the compound in this region. So this group is possibly interacting with the aromatic residues of the peripheral site through $\pi$-stacking. The LUMO energy for molecule 16 was $-0.263 \mathrm{eV}$. The electrostatic potential map (figure 4) for compound 16 indicates that a hydrogen bond type occurs between the oxygen of the acetyl group belonging to dimetoxi-phenil ring and the peripheral anionic site (attractive region, reddish) and also between the nitrogen of the enzene heterocyclic ring and the catalytic site (attractive region, reddish).

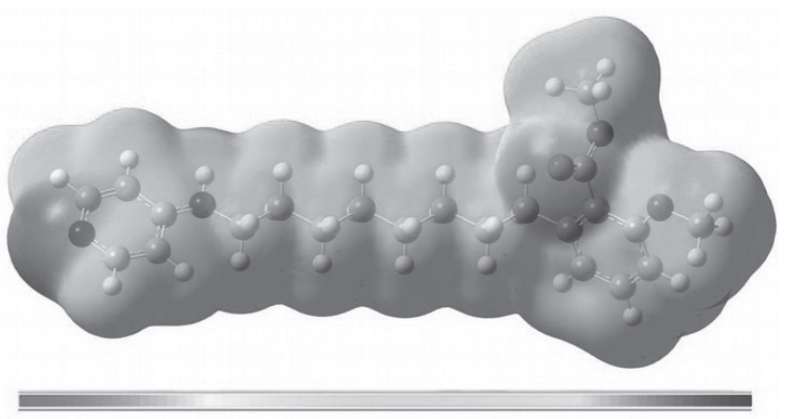

Figure 4: Molecular electrostatic potential for molecule 16.

\section{Conclusions}

This work proposes possible new inhibitor candidates of AChE designed from the anacardic acid. Based on electronic properties and also on the expected binding sites between the receptor and the ligands we concluded that molecule 16 plays the role of inhibitor better than the other structures, once PCA and HCA methods revealed that molecule 16 is very correlated to the donepezil.
Actually, one can find in the literature studies of biological activity with purified AChE, derived from the electrophorus electricus [3], indicating the therapeutic action of cholinergic compounds designed from another kind of phenolic lipid derivative. So we hope to find satisfactory experimental results of inhibition activity for our candidate.

\section{Acknowledgments}

The authors are grateful for the support given from the CAPES and CNPQ.

\section{References}

1. J. L. Cummings, Rev. Neurol. Dis. 2004, 1, 60.

2. H. Dvira, I. Silmanb, M. Harela, T. L. Rosenberryc, J. L. Sussman, Chemico-Biol Interact. 2010, 10, 187.J. L. Cummings, Rev. Neurol. Dis. 2004, 1, 60.

3. A. A. N. De Paula, J. B. L. Martins, M. L. dos Santos, L. C. Nascente, L. A. S. Romeiro, T. F. M. A. Areas, K. S. T. Vieira, N. F. Gambo, N. G. Castro, R. Gargano, Eur. J. Med. Chem. 2009, 44,3754

\section{A. S. Kiametis ${ }^{a}$, M. Abreu ${ }^{a}$, R. Gargano \& L. A. S. Romeiro ${ }^{\text {b }}$}

\footnotetext{
a University of Brasilia, Institute of Physics, CP 04454 Brasilia, Brazil ${ }^{\mathrm{b}}$ University of Brasilia, Department of Pharmacology, CP 04454 Brasilia, Brazil
} 